\title{
PDE5 Inhibitor Treatment Persistence and Adherence in Brazilian Men: Post-hoc Analyses from a 6-Month, Prospective, Observational Study
}

\author{
Carlos Cairoli', Luis Antonio Reyes ${ }^{2}$, Carsten Henneges ${ }^{3}$, Sebastian Sorsaburu ${ }^{4}$ \\ ${ }^{1}$ Hospital São Lucas, Pontifical Catholic University of Rio Grande do Sul (HSL-PUCRS), RS, Brazil; ${ }^{2}$ Eli \\ Lilly and Company, Mexico City, Mexico; ${ }^{3}$ Lilly Deutschland GmbH (CH), Bad Homburg, Germany and \\ ${ }^{4}$ Lilly Research Laboratories, Eli Lilly and Company, IN, USA
}

\section{ABSTRACT}

Purpose: Characterize persistence and adherence to phosphodiesterase type - 5 inhibitor (PDE5I) on-demand therapy over 6 months among Brazilian men in an observational, non-interventional study of Latin American men naïve to PDE5Is with erectile dysfunction (ED).

Materials and Methods: Men were prescribed PDE5Is per routine clinical practice. Persistence was defined as using $\geq 1$ dose during the previous 4 - weeks, and adherence as following dosing instructions for the most recent dose, assessed using the Persistence and Adherence Questionnaire. Other measures included the Self - Esteem and Relationship (SEAR) Questionnaire, and International Index of Erectile Function (IIEF). Multivariate logistic regression was used to identify factors associated with persistence/adherence. Results: 104 Brazilian men were enrolled; mean age by treatment was 53 to 59 years, and most presented with moderate ED (61.7\%). The prescribed PDE5I was sildenafil citrate for 50 (48.1\%), tadalafil for 36 (34.6\%), vardenafil for 15 (14.4\%), and lodenafil for 3 patients (2.9\%). Overall treatment persistence was 69.2\% and adherence was $70.2 \%$; both were numerically higher with tadalafil (75.0\%) versus sildenafil or vardenafil (range 60.0\% to 68.0\%). Potential associations of persistence and/or adherence were observed with education level, ED etiology, employment status, and coronary artery disease. Improvements in all IIEF domain scores, and both SEAR domain scores were observed for all treatments. Study limitations included the observational design, brief duration, dependence on patient self - reporting, and limited sample size.

Conclusion: Approximately two-thirds of PDE5I-naive, Brazilian men with ED were treatment persistent and adherent after 6 months. Further study is warranted to improve long-term outcomes of ED treatment.
\end{abstract}

\section{ARTICLE INFO}

Key words:

Advance Directive Adherence;

Erectile Dysfunction; sildenafil

[Supplementary Concept];

tadalafil [Supplementary

Concept]; vardenafil

[Supplementary Concept]

Int Braz J Urol. 2014; 40: 390-9

Submitted for publication:

December 13, 2013

Accepted after revision:

February 19, 2014

\section{INTRODUCTION}

Erectile dysfunction (ED) is a common condition among men worldwide and has a negative impact on interpersonal relationships, overall self - esteem, sense of well-being, and quality of life for both men and women $(1,2)$.
In a 2001 survey of more than 1,200 men aged 40 to 70 years in 9 major cities across Brazil, $46.2 \%$ reported having ED of minimal or greater severity. The prevalence of ED increased with age, and several sociological, demographic, and medical variables were also correlated with increased ED prevalence (3). 
Oral phosphodiesterase type - 5 inhibitors (PDE5Is) represent the first line of therapy for men with ED. While clinical studies have demonstrated that PDE5Is are efficacious, safe, and well tolerated, patient outcomes in clinical practice have been more variable due to a variety of factors including incorrect PDE5I administration and/or premature discontinuation of therapy $(4,5)$. Given the contribution of successful PDE5I therapy to improvements in quality of life, confidence, and relationships (5-8), it is important to understand factors that may affect patients' continuation of therapy. Continuation itself may be considered based on two aspects - "persistence" (the patient's continuation of PDE5I therapy over a prolonged duration) and "adherence" (the patient's compliance with PDE5I dosing instructions).

Recent studies examining PDE5I persistence and adherence have demonstrated widely varying rates of persistence, ranging from 29\% to $84 \%(9,10)$. Among non-persistent patients, commonly cited reasons for discontinuing PDE5I therapy have included the cost of medication, loss of interest in sex, loss of a sexual partner, lack of efficacy, or improvements in ED (10-12). Notably, a lack of efficacy may be due to either the drug or to improper use of the medication.

Given that effective PDE5I therapy requires both that patients continue to take medication and that they take it correctly, understanding factors affecting persistence and adherence in clinical practice is essential to the effective treatment of ED. In the current study, we assessed persistence and adherence to sildenafil, tadalafil, and vardenafil on-demand therapy in Brazilian men with ED and sought to identify characteristics associated with non-persistence and non-adherence. The impact of treatment on patients' ED and measures assessing satisfaction with their sexual relationships and with their ED treatment were also evaluated.

\section{PATIENTS AND METHODS}

\section{Study Design}

A 6-month, prospective, observational, non-interventional study was conducted in Brazil, Mexico, and Venezuela during the period from October 2009 through January 2011. Overall results and a detailed description of the study methods have been published previously (13). The present analysis was performed to characterize persistence and adherence specifically in the Brazilian men from that study.

In brief, sildenafil, tadalafil, vardenafil, or lodenafil on-demand dosing was prescribed to men by their clinician according to standard clinical practice. Changes to or discontinuation of the selected PDE5I treatment regimen were at the discretion of the patient and clinician. Evaluations were performed before the initiation of PDE5I therapy (baseline), and patients provided assessments of drug administration and dosing compliance, erectile function, sexual performance and satisfaction, and relationship status at 1, 3, and 6 months of treatment. The primary objective was to identify the persistence rate of prescribed PDE5I treatment at 6 months. Secondary objectives included identifying separate rates for each PDE5I and identifying factors that were associated with non-persistence and non-adherence.

\section{Patients}

Men from Brazil were eligible if they were $\geq 18$ years of age, had been clinically diagnosed with ED (of any severity or etiology), were naive to PDE5I therapy, and agreed to be prescribed PDE5I on-demand therapy. All men provided written informed consent prior to initiating the study.

\section{Outcomes Assessments}

The Persistence and Adherence Questionnaire (PAQ) was administered at 1, 3, and 6 months. The PAQ asked patients if they had taken $\geq 1$ dose of the originally prescribed PDE5I within the last 4 weeks (the persistence measure) and whether they had taken their most recent PDE5I dose according to their original treatment instructions (the adherence measure). Also administered at 1, 3, and 6 months postbaseline were the International Index of Erectile Function (IIEF) (14); the Erectile Dysfunction Inventory of Treatment Satisfaction (EDITS), a validated questionnaire (15) used to assess the patient's satisfaction with their current ED therapy; and the Self-Esteem And Relationship 
(SEAR) Questionnaire, a 14 - item validated measure (16) assessing the sexual relationship and overall confidence of the patient. Data for these measures were summarized at 6 months only for this report.

\section{Safety}

This was an observational, non-interventional study; the safety of PDE5I on-demand therapy was monitored by each patient's physician, but safety measures were not collected and analyzed for this report.

\section{Statistical analysis}

Persistence and adherence were each treated as dichotomous outcome measures. Patients were considered persistent if they had taken $\geq 1$ dose of their originally prescribed PDE5I within 4 weeks prior to a given study visit. Patients were considered adherent if they had followed treatment instructions for their most recent PDE5I dose. Patients who did not complete assessments were considered to be non-persistent and non-adherent for that visit. Patients were considered to be persistent or adherent if they met the criteria at all prior postbaseline assessments. Treatment groups correspond to the PDE5I initially prescribed.

Analyses were performed as described previously (13). The primary analysis was the calculation of the proportion of patients who met the criteria for persistence at 6 months. Persistence was also assessed at 3 months and adherence at 1,3 , and 6 months. Persistence and adherence rates were also calculated for each PDE5I (sildenafil, tadalafil, vardenafil, and lodenafil) as well as a corresponding 95\% confidence interval (CI), which was calculated as an exact CI for the binomial proportion using the $\mathrm{F}$ distribution. To identify factors associated with persistence and adherence, a multivariate logistic regression model was used to analyze data for the complete study population from Brazil, Mexico and Venezuela. Factors significantly associated with outcomes at the 10\% level were included in the model, with stepwise reduction to exclude factors with $p$ value $>0.05$. Identification of factors presented in this report was based on modeling from the overall study po- pulation applied to data for patients from Brazil only. The Spearman Rank correlation and Kruskal-Wallis test were used to investigate associations between patient characteristics and adherence/ persistence.

Descriptive statistics (e.g., mean, standard deviation [SD], median, 95\% CI) were used to summarize results from the IIEF, EDITS, and SEAR. Treatment groups correspond to the PDE5I initially prescribed. Findings were considered statistically significant if $p<0.05$. Due to the small sample size, which limits statistical power, results with $p \geq 0.05$ and $<0.1$ were considered of potential interest and reported here as "weakly significant."

\section{RESULTS}

\section{Patients}

A total of 104 Brazilian patients entered the study; the prescribed PDE5I was sildenafil citrate for 50 (48.1\%); tadalafil for 36 (34.6\%); vardenafil for 15 (14.4\%), and lodenafil for 3 patients (2.9\%). For lodenafil, data were generally presented in the tables but not discussed in comparison to other treatment groups due the lack of ability to draw inferences due to the very low sample size. Of all men, 90 (86.5\%) completed treatment, with comparable rates of completion across the three commonly prescribed treatments (84.0 to $88.9 \%$ ).

Patient characteristics are summarized by study treatment in Table-1. Mean (SD) ages ranged from 52.7 (17.9) to 58.7 (9.7) years, with an overall range of 26.0 to 79.0 years. Overall, most patients had ED of mixed (47.1\%) or organic (36.3\%) etiology, and for the majority (61.7\%), ED severity was moderate. A slight majority of patients were diagnosed with hypertension (51.9\%), 24\% had diabetes mellitus, and 9.6\% were obese.

\section{Persistence and Adherence - Overall Study Po- pulation and Treatment Groups}

Rates of treatment persistence and adherence are shown in Table-2, and treatment persistence is illustrated by treatment in Figure-1A. At 6 months, the overall rate of treatment persistence was $69.2 \%$. Treatment persistence was highest with tadalafil (75.0\%), but similar between sildenafil and vardenafil (64.0\% and 66.7\%, respectively). Persistence at 
Table 1 - Demographics and Baseline Characteristics.

\begin{tabular}{|c|c|c|c|c|c|}
\hline & $\begin{array}{l}\text { Tadalafil } \\
(\mathrm{N}=36)\end{array}$ & $\begin{array}{l}\text { Sildenafil } \\
(N=50)\end{array}$ & $\begin{array}{l}\text { Vardenafil } \\
(N=15)\end{array}$ & $\begin{array}{l}\text { Lodenafil } \\
\qquad(\mathrm{N}=3)\end{array}$ & $\begin{array}{l}\text { Overall } \\
(N=104)\end{array}$ \\
\hline Mean age (SD), years & $58.7(9.7)$ & $58.3(9.9)$ & $55.3(15.4)$ & $52.7(17.9)$ & $57.8(10.9)$ \\
\hline Mean BMI (SD), kg/m² & $27.2(4.2)$ & $27.8(5.5)$ & $25.9(6.0)$ & $25.0(2.8)$ & $27.3(5.1)$ \\
\hline \multicolumn{6}{|l|}{ Education level, n (\%) } \\
\hline Primary education & $13(36.1)$ & $13(27.1)$ & $7(46.7)$ & $1(33.3)$ & $34(33.3)$ \\
\hline Secondary education & $13(36.1)$ & $19(39.6)$ & $5(33.3)$ & $0(0)$ & $37(36.3)$ \\
\hline Tertiary education & $5(13.9)$ & $7(14.6)$ & $1(6.7)$ & $1(33.3)$ & $14(13.7)$ \\
\hline University education & $4(11.1)$ & $8(16.7)$ & $1(6.7)$ & $1(33.3)$ & $14(13.7)$ \\
\hline Postgraduate education & $1(2.8)$ & $1(2.1)$ & $0(0)$ & $0(0)$ & $2(2.0)$ \\
\hline Median ED duration, years $(Q 1, Q 3)^{a}$ & $2.2(1.3,3.0)$ & $3.0(2.0,3.1)$ & $0.8(0.6,1.0)$ & $0.8(0.8,0.8)$ & $2.0(1.1,3.0)$ \\
\hline \multicolumn{6}{|l|}{ ED Severity Categories, $\mathbf{n}(\%)$} \\
\hline Mild & $3(9.1)$ & $7(16.3)$ & $2(13.3)$ & $1(33.3)$ & $13(13.8)$ \\
\hline Moderate & $18(54.5)$ & $28(65.1)$ & $10(66.7)$ & $2(66.7)$ & $58(61.7)$ \\
\hline Severe & $12(36.4)$ & $8(18.6)$ & $3(20.0)$ & $0(0)$ & $23(24.5)$ \\
\hline \multicolumn{6}{|l|}{ ED Etiology, n (\%) } \\
\hline Mixed & $19(52.8)$ & $21(43.8)$ & $8(53.3)$ & $0(0)$ & $48(47.1)$ \\
\hline Organic & $13(36.1)$ & $17(35.4)$ & $6(40.0)$ & $1(33.3)$ & $37(36.3)$ \\
\hline Psychogenic & $4(11.1)$ & $9(18.8)$ & $1(6.7)$ & $2(66.7)$ & $16(15.7)$ \\
\hline \multicolumn{6}{|l|}{ Pre-existing Conditions, $n$ (\%) yes } \\
\hline Hypertension & $19(52.8)$ & $25(50.0)$ & $8(53.3)$ & $2(66.7)$ & $54(51.9)$ \\
\hline Diabetes Mellitus & $7(19.4)$ & $14(28.0)$ & $4(26.7)$ & $0(0)$ & $25(24.0)$ \\
\hline Obesity & $6(16.7)$ & $3(6.0)$ & $1(6.7)$ & $0(0)$ & $10(9.6)$ \\
\hline Coronary Artery Disease & $1(2.8)$ & $3(6.0)$ & $0(0)$ & $0(0)$ & $4(3.8)$ \\
\hline Benign Prostatic Hyperplasia & $2(5.6)$ & $3(6.0)$ & $2(13.3)$ & $0(0)$ & $7(6.7)$ \\
\hline Lower Urinary Tract Symptoms & $2(5.6)$ & $3(6.0)$ & $0(0)$ & $0(0)$ & $5(4.8)$ \\
\hline Hyperlipidemia & $6(16.7)$ & $5(10.2)$ & $2(13.3)$ & $0(0)$ & $13(12.6)$ \\
\hline
\end{tabular}

$\mathbf{B M I}=$ body mass index; $\mathbf{E D}=$ erectile dysfunction; $\mathbf{N}=$ number of patients in treatment group; $\mathbf{n}=$ number of patients observed/reported; $\mathbf{S D}=$ standard deviation $\mathbf{Q}=$ quartile.

${ }^{\text {aED }}$ duration data were available for: Tadalafil, $n=10$; sildenafil, $n=18$; vardenafil, $n=2$; lodenafil, $n=1$; overall, $n=31$. 
3 months was also highest with tadalafil (86.1\%) but similar for sildenafil (70.0\%) and vardenafil (73.3\%).

Overall adherence was $70.2 \%$ at 6 months (Table-2). The rate of adherence was highest with tadalafil (75.0\%), then sildenafil (68.0\%) and vardenafil (60.0\%) (Table-2, Figure-1B). At 1 and 3 months, adherence rates were also highest in tadalafil patients (Figure-1B).

The differences in persistence and adherence estimates for the PDE5Is were not significant, as indicated by the overlapping 95\% CIs.

Table 2 - Treatment Persistence and Adherence.

\begin{tabular}{|c|c|c|c|c|c|c|c|c|c|c|}
\hline \multirow[b]{2}{*}{ Parameter } & \multicolumn{2}{|c|}{$\begin{array}{l}\text { Tadalafil } \\
(\mathrm{N}=36)\end{array}$} & \multicolumn{2}{|r|}{$\begin{array}{l}\text { Sildenafil } \\
(N=50)\end{array}$} & \multicolumn{2}{|c|}{$\begin{array}{l}\text { Vardenafil } \\
(N=15)\end{array}$} & \multicolumn{2}{|r|}{$\begin{array}{l}\text { Lodenafil } \\
\qquad(\mathrm{N}=3)\end{array}$} & \multicolumn{2}{|c|}{$\begin{array}{c}\text { Overall } \\
(\mathrm{N}=104)\end{array}$} \\
\hline & $n$ & $\begin{array}{c}\% \\
(95 \% \mathrm{Cl})\end{array}$ & $\mathrm{n}$ & $\begin{array}{c}\% \\
(95 \% \mathrm{Cl})\end{array}$ & $\mathrm{n}$ & $\begin{array}{c}\% \\
(95 \% \mathrm{Cl})\end{array}$ & $\mathrm{n}$ & $\begin{array}{c}\% \\
(95 \% \mathrm{Cl})\end{array}$ & $\mathrm{n}$ & $\begin{array}{c}\% \\
(95 \% \mathrm{Cl})\end{array}$ \\
\hline Persistence at 3 months & 31 & $\begin{array}{c}86.1 \\
(70.5,95.3)\end{array}$ & 35 & $\begin{array}{c}70.0 \\
(55.4,82.1)\end{array}$ & 11 & $\begin{array}{c}73.3 \\
(44.9,92.2)\end{array}$ & 3 & $\begin{array}{c}100 \\
(29.2,100)\end{array}$ & 80 & $\begin{array}{c}76.9 \\
(67.6,84.6)\end{array}$ \\
\hline Persistence at 6 months & 27 & $\begin{array}{c}75.0 \\
(57.8,87.9)\end{array}$ & 32 & $\begin{array}{c}64.0 \\
(49.2,77.1)\end{array}$ & 10 & $\begin{array}{c}66.7 \\
(38.4,88.2)\end{array}$ & 3 & $\begin{array}{c}100 \\
(29.2,100)\end{array}$ & 72 & $\begin{array}{c}69.2 \\
(59.4,77.9)\end{array}$ \\
\hline Adherence at 1 month & 33 & $\begin{array}{c}91.7 \\
(77.5,98.2)\end{array}$ & 39 & $\begin{array}{c}78.0 \\
(64.0,88.5)\end{array}$ & 12 & $\begin{array}{c}80.0 \\
(51.9,95.7)\end{array}$ & 3 & $\begin{array}{c}100 \\
(29.2,100)\end{array}$ & 87 & $\begin{array}{c}83.7 \\
(75.1,90.2)\end{array}$ \\
\hline Adherence at 3 months & 30 & $\begin{array}{c}83.3 \\
(67.2,93.6)\end{array}$ & 35 & $\begin{array}{c}70.0 \\
(55.4,82.1)\end{array}$ & 11 & $\begin{array}{c}73.3 \\
(44.9,92.2)\end{array}$ & 3 & $\begin{array}{c}100 \\
(29.2,100)\end{array}$ & 79 & $\begin{array}{c}76.0 \\
(66.6,83.8)\end{array}$ \\
\hline Adherence at 6 months & 27 & $\begin{array}{c}75.0 \\
(57.8,87.9)\end{array}$ & 34 & $\begin{array}{c}68.0 \\
(53.3,80.5)\end{array}$ & 9 & $\begin{array}{c}60.0 \\
(32.3,83.7)\end{array}$ & 3 & $\begin{array}{c}100 \\
(29.2,100)\end{array}$ & 73 & $\begin{array}{c}70.2 \\
(60.4,78.8)\end{array}$ \\
\hline
\end{tabular}

$\mathbf{N}=$ overall number of patients in treatment group; $\mathbf{n}=$ number of patients who met the criteria for indicated parameter.

Note: Additionally, persistent but not adherent patients at 3 months included 2 tadalafil, 3 sildenafil, and 1 vardenafil patient, and at 6 months included 2 tadalafil, 2 sildenafil, and 1 vardenafil patient.

Figure 1 - Treatment persistence and adherence by treatment group for sildenafil, tadalafil, and vardenafil. A) Treatment persistence by treatment group at 3 and 6 months, B) Treatment adherence at 1, 3, and 6 months.
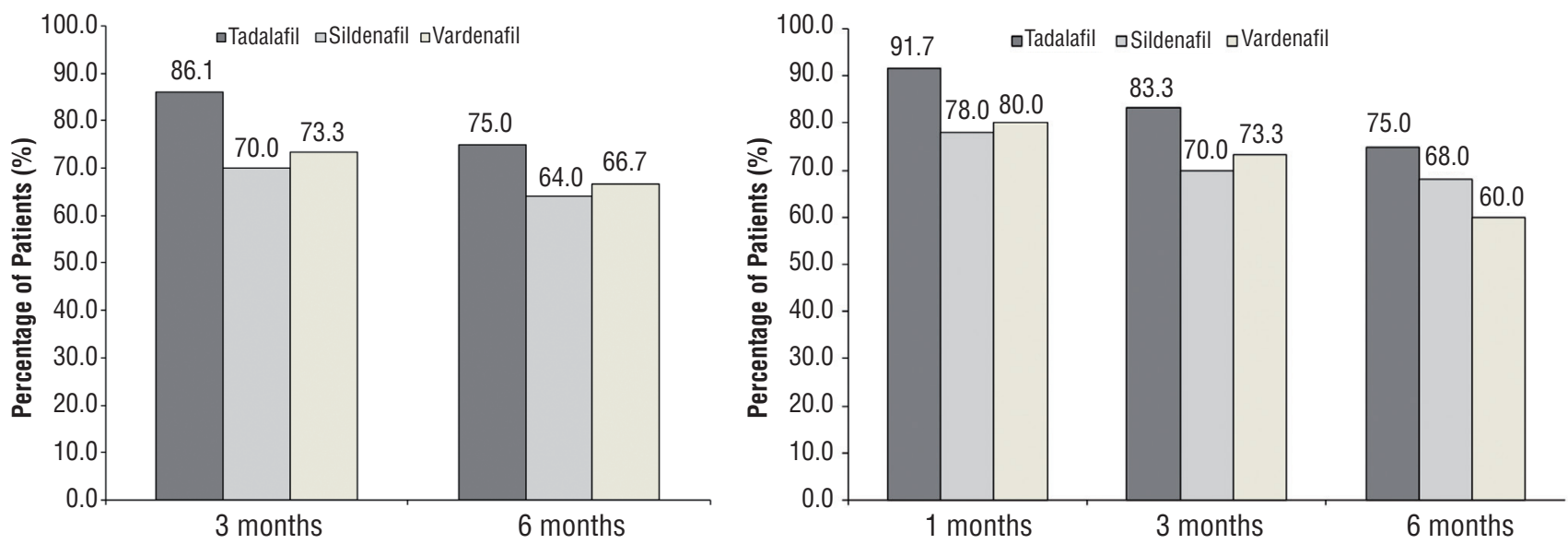
Baseline Characteristics of Patients by 6-Month Persistence and Adherence Status

Men who were persistent at 6 months differed from men who were non-persistent in that on average they were slightly younger (mean age 57.0 [11.2] versus 59.5 [10.2] years) and had a shorter median duration of ED (2.0 versus 3.0 years; Table-3). As shown in Table-3, other factors that appeared to

Table 3 - Baseline Characteristics by 6-month Treatment Persistence and Adherence.

\begin{tabular}{|c|c|c|c|c|}
\hline & $\begin{array}{c}\text { Persistent } \\
(\mathrm{N}=72)\end{array}$ & $\begin{array}{c}\text { Non-Persistent } \\
(\mathrm{N}=32)\end{array}$ & $\begin{array}{l}\text { Adherent } \\
(\mathrm{N}=73)\end{array}$ & $\begin{array}{c}\text { Non-adherent } \\
(\mathrm{N}=31)\end{array}$ \\
\hline Mean age (SD), years & $57.0(11.2)$ & $59.5(10.2)$ & $57.2(10.9)$ & $59.3(11.0)$ \\
\hline \multicolumn{5}{|l|}{ Education level, n (\%) } \\
\hline Primary education & $21(29.2)$ & $13(43.3)$ & $22(30.1)$ & $12(41.4)$ \\
\hline Secondary education & $25(34.7)$ & $12(40.0)$ & $24(32.9)$ & $13(44.8)$ \\
\hline Tertiary education & $10(13.9)$ & $4(13.3)$ & $12(16.4)$ & $2(6.9)$ \\
\hline University education & $14(19.4)$ & $0(0)$ & $13(17.8)$ & $1(3.4)$ \\
\hline Postgraduate education & $2(2.8)$ & $0(0)$ & $2(2.7)$ & $0(0)$ \\
\hline \multicolumn{5}{|l|}{ Employment status, n (\%) } \\
\hline Full-time & $44(61.1)$ & $11(37.9)$ & $45(61.6)$ & $10(35.7)$ \\
\hline Part-time & $4(5.6)$ & $5(17.2)$ & $3(4.1)$ & $6(21.4)$ \\
\hline Retired & $17(23.6)$ & $10(34.5)$ & $18(24.7)$ & $9(32.1)$ \\
\hline Unemployed & $7(9.7)$ & $3(10.3)$ & $7(9.6)$ & $3(10.7)$ \\
\hline \multicolumn{5}{|l|}{ Race, n (\%) } \\
\hline Black or African America & $8(11.1)$ & $6(18.8)$ & $8(11.0)$ & $6(19.4)$ \\
\hline White & $64(88.9)$ & $26(81.3)$ & $65(89.0)$ & $25(80.6)$ \\
\hline Median ED duration, years $(Q 1, Q 3)^{\mathrm{a}}$ & $2.0(1.1,3.0)$ & $3.0(2.0,4.0)$ & $2.0(1.1,3.0)$ & $3.0(2.0,4.0)$ \\
\hline \multicolumn{5}{|l|}{ ED severity, n (\%) } \\
\hline Mild & $11(15.9)$ & $2(8.0)$ & $10(14.1)$ & $3(13.0)$ \\
\hline Moderate & $38(55.1)$ & $20(80.0)$ & $43(60.6)$ & $15(65.2)$ \\
\hline Severe & $20(29.0)$ & $3(12.0)$ & $18(25.4)$ & $5(21.7)$ \\
\hline \multicolumn{5}{|l|}{ ED etiology, $n(\%)$} \\
\hline Mixed & $30(41.7)$ & $18(60.0)$ & $30(41.1)$ & $18(62.1)$ \\
\hline Organic & $26(36.1)$ & $11(36.7)$ & $27(37.0)$ & $10(34.5)$ \\
\hline Psychogenic & $15(20.8)$ & $1(3.3)$ & $15(20.5)$ & $1(3.4)$ \\
\hline \multicolumn{5}{|l|}{ Comorbid conditions, n (\%) yes } \\
\hline Hypertension & $34(47.2)$ & $20(62.5)$ & $36(49.3)$ & $18(58.1)$ \\
\hline Diabetes Mellitus & $15(20.8)$ & $10(31.3)$ & $15(20.5)$ & $10(32.3)$ \\
\hline Coronary Artery Disease & $1(1.4)$ & $3(9.4)$ & $0(0)$ & $4(12.9)$ \\
\hline
\end{tabular}

$\overline{\mathbf{E D}}=$ erectile dysfunction; $\mathbf{N}=$ number of patients in treatment group; $\mathbf{n}=$ number of patients observed/reported; $\mathbf{S D}=$ standard deviation. aED duration data were available for: Tadalafil, $\mathbf{n}=10$; sildenafil, $\mathbf{n}=18$; vardenafil, $\mathbf{n}=2$; 
differ between persistent and non-persistent men included employment status, educational level, and the presence of diabetes mellitus, hypertension, and coronary artery disease. The severity of ED differed in that a greater proportion of men who were persistent (versus non-persistent) had severe ED (29.0\% versus $12.0 \%$ ) and a lower proportion had moderate ED (55.1\% versus 80.0\%). The etiology of ED also differed by up to $18.3 \%$ between persistent and non-persistent men. Characteristics associated with adherence versus non-adherence were generally closely comparable to those associated with persistence versus non-persistence.

Correlation Analysis of Factors Associated with 6-month Persistence and Adherence Status

Results of analyses of the correlation of various factors with persistence or adherence at 6-months are shown in Table-4. For persistence, no correlations achieved statistical significance; however, weakly significant p-values $(0.05 \leq \mathrm{p}<$ $0.10)$ were seen for education level $(p=0.065)$ and coronary artery disease $(p=0.051)$, while $p$-values for employment status $(p=0.108)$ and ED etiology $(p=0.111)$ approached this level.

Statistically significant associations with adherence were seen for employment status ( $\mathrm{p}=$ $0.022)$ and for coronary artery disease $(p=0.002)$. Weak statistical significance was seen for education level $(p=0.097)$, while ED etiology approached weak statistical significance $(p=0.103)$.

\section{Therapeutic Efficacy - Erectile Function, Sexual} Performance, and Relationship Status

Sildenafil, tadalafil, and vardenafil on-demand dosing for 6 months were all effective in treating ED in Brazilian men, as baseline-to-endpoint improvements were observed for all treatments in

Table 4 - Correlation of Factors with Treatment Persistence and Adherence.

\begin{tabular}{|c|c|c|c|c|}
\hline \multirow[b]{2}{*}{ Factor } & \multicolumn{2}{|c|}{ Persistence } & \multicolumn{2}{|c|}{ Adherence } \\
\hline & Correlation coefficient & P-value ${ }^{a}$ & Correlation coefficient & P-value ${ }^{a}$ \\
\hline Age $^{a}$ & -0.110 & 0.268 & -0.104 & 0.292 \\
\hline Marital status ${ }^{\mathrm{b}}$ & $\mathrm{N} / \mathrm{A}$ & 0.279 & $\mathrm{~N} / \mathrm{A}$ & 0.131 \\
\hline Education level $\left.\right|^{b}$ & $\mathrm{~N} / \mathrm{A}$ & 0.065 & $\mathrm{~N} / \mathrm{A}$ & 0.097 \\
\hline Employment status $^{b}$ & $\mathrm{~N} / \mathrm{A}$ & 0.108 & $\mathrm{~N} / \mathrm{A}$ & 0.022 \\
\hline Race $^{\mathrm{b}}$ & $\mathrm{N} / \mathrm{A}$ & 0.294 & $N / A$ & 0.253 \\
\hline ED severity ${ }^{\mathrm{a}}$ & 0.076 & 0.464 & 0.020 & 0.848 \\
\hline ED etiology ${ }^{b}$ & $\mathrm{~N} / \mathrm{A}$ & 0.111 & $\mathrm{~N} / \mathrm{A}$ & 0.103 \\
\hline Duration of $\mathrm{ED}^{\mathrm{a}}$ & -0.234 & 0.205 & -0.207 & 0.264 \\
\hline Diabetes mellitus ${ }^{\mathrm{a}}$ & -0.113 & 0.255 & -0.125 & 0.205 \\
\hline Dyslipidemia $^{a}$ & 0.058 & 0.559 & -0.014 & 0.890 \\
\hline Hypertension $^{\mathrm{a}}$ & -0.141 & 0.153 & -0.080 & 0.419 \\
\hline Coronary artery disease ${ }^{a}$ & -0.192 & 0.051 & -0.307 & 0.002 \\
\hline Depressiona & 0.077 & 0.437 & -0.018 & 0.860 \\
\hline Initial treatment ${ }^{b}$ & $\mathrm{~N} / \mathrm{A}$ & 0.465 & $\mathrm{~N} / \mathrm{A}$ & 0.474 \\
\hline
\end{tabular}

ED $=$ erectile dysfunction .

${ }^{a}$ Spearman correlation coefficient, used when both variables were continuous, binary or ordered categorical variables; bKruskal-Wallis test, used when one variable was a continuous, binary, or ordered categorical variable and another was not an ordered categorical variable. 
all IIEF domain scores and both SEAR domain scores (Table-5). Patients also reported high levels of treatment satisfaction based on EDITS scores at 6 months ranging from $65.6 \%$ with vardenafil to $82.2 \%$ with tadalafil.

\section{Safety}

This was an observational, non-interventional study; therefore patient safety was monitored by physicians at each respective study site, but safety measures were not collected and analyzed for this report.

\section{DISCUSSION}

Among the 104 Brazilian men with ED who entered this study, 69.2\% $(n=72)$ were persistent after 6 months of PDE5I therapy, while $70.2 \%(n=73)$ were considered adherent through
6 months (Table-2). Patients in the tadalafil treatment group exhibited the highest rates of persistence and adherence through 6 months (75.0\% for both), with somewhat lower rates for sildenafil and vardenafil (persistence, 64.0\% - 66.7\%; adherence, $60.0 \%$ - 68.0\%).

Comparing baseline characteristics based on persistence and non-persistence at 6 months, men who were subsequently non-persistent were slightly younger, and had a shorter duration of ED. The proportion of patients with severe ED was greater, and the proportion with ED of moderate severity was lower, for persistent versus non-persistent men. Mixed etiology was more prevalent and psychogenic etiology less prevalent in persistent versus non-persistent men. Persistent men also had more full-time employment, higher average educational level, and a higher proportion had diabetes mellitus, hypertension, or coronary

Table 5 - Measures of Therapeutic Effectiveness - Results for IIEF, EDITS, and SEAR at Baseline and 6 Months.

\begin{tabular}{|c|c|c|c|c|c|c|c|c|}
\hline \multirow{2}{*}{$\begin{array}{l}\text { Domain } \\
\text { Parameter, } \\
\text { mean (SD) }\end{array}$} & \multicolumn{2}{|c|}{ Tadalafil } & \multicolumn{2}{|c|}{ Sildenafil } & \multicolumn{2}{|c|}{ Vardenafil } & \multicolumn{2}{|c|}{ Lodenafil } \\
\hline & Baseline & $\begin{array}{l}\text { Change from } \\
\text { baseline }^{\mathrm{a}}\end{array}$ & Baseline & $\begin{array}{l}\text { Change from } \\
\text { baseline }\end{array}$ & Baseline & $\begin{array}{l}\text { Change from } \\
\text { baseline }^{a}\end{array}$ & Baseline & $\begin{array}{l}\text { Change } \\
\text { from } \\
\text { baseline }\end{array}$ \\
\hline $\begin{array}{l}\text { IIEF Erectile } \\
\text { Function }\end{array}$ & $\begin{array}{l}12.5 \\
(5.7)\end{array}$ & $10.1(8.5)$ & $13.4(6.8)$ & $8.4(8.2)$ & $11.5(6.4)$ & $8.4(6.6)$ & $19.3(6.1)$ & $7.7(6.0)$ \\
\hline $\begin{array}{l}\text { IIEF Intercourse } \\
\text { Satisfaction }\end{array}$ & $5.3(3.2)$ & $4.8(4.8)$ & $6.2(3.8)$ & $3.2(4.9)$ & $5.5(3.3)$ & $3.5(3.7)$ & $9.3(4.0)$ & $2.7(4.2)$ \\
\hline $\begin{array}{l}\text { IIEF Orgasmic } \\
\text { Function }\end{array}$ & $5.8(3.8)$ & $2.2(3.7)$ & $5.6(3.4)$ & $1.8(3.3)$ & $5.5(3.5)$ & $1.0(3.3)$ & $7.3(2.1)$ & $2.3(2.5)$ \\
\hline $\begin{array}{l}\text { IIEF Sexual } \\
\text { Desire }\end{array}$ & $6.5(1.8)$ & $0.7(2.3)$ & $6.8(2.3)$ & $0.4(2.3)$ & $5.7(2.1)$ & $0.1(2.2)$ & $7.0(2.0)$ & $1.3(1.2)$ \\
\hline $\begin{array}{l}\text { IIEF Overall } \\
\text { Satisfaction }\end{array}$ & $4.6(2.0)$ & $3.3(2.4)$ & $4.8(2.3)$ & $2.5(3.3)$ & $4.6(2.1)$ & $1.6(2.1)$ & $5.3(2.3)$ & $4.0(2.7)$ \\
\hline $\begin{array}{l}\text { SEAR Sexual } \\
\text { Relationship }\end{array}$ & $\begin{array}{c}41.9 \\
(24.7)\end{array}$ & $31.2(31.3)$ & $\begin{array}{l}51.7 \\
(19.1)\end{array}$ & $21.3(25.3)$ & $\begin{array}{l}44.8 \\
(24.8)\end{array}$ & $19.2(20.8)$ & $67.7(23.7)$ & $\begin{array}{c}20.8 \\
(23.5)\end{array}$ \\
\hline $\begin{array}{l}\text { SEAR } \\
\text { Confidence }\end{array}$ & $\begin{array}{c}41.6 \\
(22.4)\end{array}$ & 33.3 (31.6) & $\begin{array}{c}47.7 \\
(22.8)\end{array}$ & $24.9(28.2)$ & $\begin{array}{c}48.6 \\
(18.9)\end{array}$ & $14.7(18.9)$ & $61.1(18.8)$ & $\begin{array}{c}26.4 \\
(23.0)\end{array}$ \\
\hline $\begin{array}{l}\text { EDITS Total } \\
\text { (Score) }\end{array}$ & $N / A$ & $82.2(23.7)^{\mathrm{a}}$ & $\mathrm{N} / \mathrm{A}$ & $78.1(22.9)^{\mathrm{a}}$ & $\mathrm{N} / \mathrm{A}$ & $65.6(30.3)^{\mathrm{a}}$ & $N / A$ & $91.7(8.0)^{\mathrm{a}}$ \\
\hline
\end{tabular}

EDITS = Erectile Dysfunction Inventory of Treatment Satisfaction; IIEF = International Index of Erectile Function; $\mathbf{S D}=$ standard deviation; SEAR = Self-Esteem and Relationship questionnaire. aPatients with data available both at baseline and 6 months only, except EDITS score; ${ }^{b}$ As EDITS scores are not collected prior to treatment, data show scores at 6 months, rather than change from baseline. 
artery disease. Findings for adherence were generally consistent with those for persistence.

Correlation analyses identified no statistically significant correlations with persistence; however, borderline/weakly significant correlations were observed for education level and coronary artery disease, while employment status and ED etiology had p-values just above the threshold to be considered weakly significant. Statistically significant correlations with adherence were seen for employment status and coronary artery disease, with a weakly significant correlation for education level, and ED etiology just above the threshold for weak significance. While borderline/weakly significant findings were not considered statistically significant, they could suggest areas of interest for future clinical studies or patient populations that may require additional care to improve treatment success in clinical practice.

Notably, rates of persistence and adherence were numerically higher with tadalafil versus sildenafil or vardenafil. While the reasons for this finding are unclear, there was numerically greater improvement observed with tadalafil than with sildenafil or vardenafil for most efficacy measures, including the SEAR relationship and self-confidence domains, and the various IIEF domains (including erectile function). Although modest differences in efficacy must be interpreted with caution, patients experiencing better results may have been more likely to continue therapy. However, baseline differences between patients receiving the various treatments could also have contributed to these findings.

The rates of persistence and adherence observed here are difficult to compare directly to some previous reports due to use of chart reviews, differences in follow-up times, or other methodological differences $(9,11,17)$. Nonetheless, the findings are consistent with a persistence rate of 69\% (per at least one prescription refill) through 6 months found in patients who began successful therapy with sildenafil (12), and perhaps with discontinuation rates of $47.6 \%$ and 45.4\% among men previously treated and men naive to ED therapy, respectively, after a mean of 1718 months follow-up (18), although higher persistence was reported at 6 and 12 months with tadalafil in one study (90\% and 84\%, respectively) (10).
In addition to the different numbers of patients assigned to each treatment and differences in some patient characteristics between treatments, other limitations of this study should be considered when interpreting these results. First, as a non-interventional, observational study, conditions of patient and treatment selection, and monitoring of follow-up were less stringent controlled than in a randomized clinical trial, and some factors influencing self-reporting could have differed between study sites. Second, some factors that could be associated with PDE5I persistence/adherence were not included in the analyses, such as the effects of concomitant medications. Third, the sample size was relatively limited, and may not be representative of Brazilian men seeking treatment for ED overall. Finally, the 6-month observational period for this study was relatively brief in comparison to other studies examining treatment compliance, which often have had observation periods $\geq 1$ year; thus, the results may be less representative of prolonged PDE5I therapy. Additional study is warranted to further examine PDE5I persistence and adherence in Brazilian men, as well as to further investigate the predictive value of factors correlated with persistence and adherence. Future studies could include larger sample sizes, treatment randomization, regimented monitoring, and observational periods $\geq 1$ year.

\section{CONCLUSIONS}

In this observational study, $69.2 \%$ of PDE5I-naive Brazilian men were treatment persistent with sildenafil, tadalafil, vardenafil, or lodenafil on-demand dosing after 6 months, while $70.2 \%$ were treatment adherent. Men who were initially prescribed tadalafil demonstrated somewhat, but not significantly, higher persistence and adherence rates than those who were prescribed sildenafil or vardenafil. Patient characteristics that showed some degree of association with higher persistence and/or adherence rates included higher education level, lack of coronary artery disease, full-time employment, ED of psychogenic origin, and ED of a shorter duration since diagnosis. 


\section{CONFLICT OF INTEREST}

\author{
Company \\ The study was funded by Eli Lilly and
}

Drs. Luis Antonio Reyes, Carsten Henneges and Sebastian Sorsaburu are employees of Eli Lilly and Company

Other authors have no conflict of interests

\section{REFERENCES}

1. Chen KK, Jiann BP, Lin JS, Lee SS, Huang ST, Wang CJ, et al.: Efficacy and safety of on-demand oral tadalafil in the treatment of men with erectile dysfunction in Taiwan: a randomized, double-blind, parallel, placebo-controlled clinical study. J Sex Med. 2004; 1: 201-8. Erratum in: J Sex Med. 2005; 2: 158.

2. Rodríguez Vela L, Lledó García E, Rajmil 0, Mo D, Cassinello A, Casariego J: Tadalafil vs sildenafil patient preference in Spanish men with erectile dysfunction: results from an International Multicentric Study. Actas Urol Esp. 2006; 30: 67-79.

3. Moreira ED Jr, Abdo CH, Torres EB, Lôbo CF, Fittipaldi JA: Prevalence and correlates of erectile dysfunction: results of the Brazilian study of sexual behavior. Urology. 2001; 58: 583-8.

4. Sadovsky R, Brock GB, Gray M, Jensen PK, Gutkin SW, Sorsaburu S: Optimizing treatment outcomes with phosphodiesterase type 5 inhibitors for erectile dysfunction: opening windows to enhanced sexual function and overall health. J Am Acad Nurse Pract. 2011; 23: 320-30.

5. Sato Y, Tanda H, Kato S, Onishi S, Nitta T, Koroku M: How long do patients with erectile dysfunction continue to use sildenafil citrate? Dropout rate from treatment course as outcome in real life. Int J Urol. 2007; 14: 339-42; discussion 343.

6. Fisher WA, Rosen RC, Mollen M, Brock G, Karlin G, Pommerville $P$, et al.: Improving the sexual quality of life of couples affected by erectile dysfunction: a double-blind, randomized, placebo-controlled trial of vardenafil. J Sex Med. 2005; 2: 699-708.

7. Rosen R, Goldstein I, Huang XY, Bangerter K, Taylor T: The Treatment Satisfaction Scale (TSS) is a sensitive measure of treatment effectiveness for both patients and partners: results of a randomized controlled trial with vardenafil. J Sex Med. 2007; 4: 1009-21.

8. Seftel AD, Buvat J, Althof SE, McMurray JG, Zeigler HL, Burns $\mathrm{PR}$, et al.: Improvements in confidence, sexual relationship and satisfaction measures: results of a randomized trial of tadalafil $5 \mathrm{mg}$ taken once daily. Int J Impot Res. 2009; 21: 240-8.
9. Mulhall JP, McLaughlin TP, Harnett JP, Scott B, Burhani S, Russell D: Medication utilization behavior in patients receiving phosphodiesterase type 5 inhibitors for erectile dysfunction. J Sex Med. 2005; 2: 848-55.

10. Roumeguère $T$, Verheyden $B$, Arver $S$, Bitton $A$, Belger $M$, Schmitt $\mathrm{H}$; et al.: Therapeutic response after first month of tadalafil treatment predicts 12 months treatment continuation in patients with erectile dysfunction: results from the DETECT study. J Sex Med. 2008; 5; 1708-19.

11. Jiann BP, Yu CC, Su CC, Tsai JY: Compliance of sildenafil treatment for erectile dysfunction and factors affecting it. Int J Impot Res. 2006; 18: 146-9.

12. Klotz T, Mathers M, Klotz R, Sommer F: Why do patients with erectile dysfunction abandon effective therapy with sildenafil (Viagra)? Int J Impot Res. 2005; 17: 2-4.

13. Rubio-Aurioles E, Reyes LA, Borregales L, Cairoli C, Sorsaburu S: A 6 month, prospective, observational study of PDE5 inhibitor treatment persistence and adherence in Latin American men with erectile dysfunction. Curr Med Res Opin. 2013; 29: 695-706.

14. Rosen RC, Riley A, Wagner G, Osterloh IH, Kirkpatrick $J$, Mishra $A$ : The international index of erectile function (IIEF): a multidimensional scale for assessment of erectile dysfunction. Urology. 1997; 49: 822-30.

15. Althof SE, Corty EW, Levine SB, Levine F, Burnett AL, McVary $K$, et al.: EDITS: development of questionnaires for evaluating satisfaction with treatments for erectile dysfunction. Urology. 1999; 53: 793-9.

16. Cappelleri JC, Althof SE, Siegel RL, Shpilsky A, Bell SS, Duttagupta S: Development and validation of the SelfEsteem And Relationship (SEAR) questionnaire in erectile dysfunction. Int J Impot Res. 2004; 16: 30-8.

17. Gonzalgo ML, Brotzman M, Trock BJ, Geringer AM, Burnett $A L$, Jarow JP: Clinical efficacy of sildenafil citrate and predictors of long-term response. J Urol. 2003; 170: 503-6.

18. Souverein PC, Egberts AC, Meuleman EJ, Urquhart J, Leufkens HG: Incidence and determinants of sildenafil (dis) continuation: the Dutch cohort of sildenafil users. Int J Impot Res. 2002; 14: 259-65.

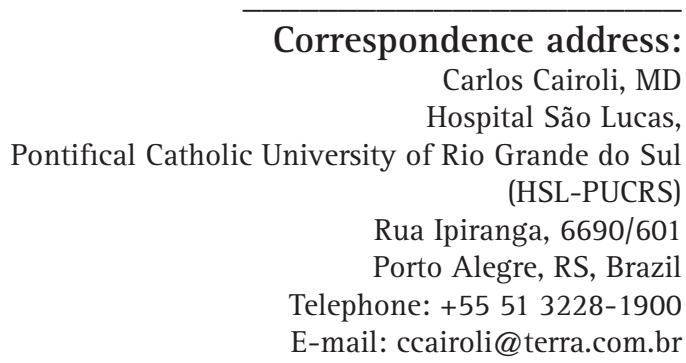

